\title{
Therapeutic apheresis: Technical modalities and therapeutic applications
}

\author{
Grapsa Eirini ${ }^{1}$ and Panokostas Dimitrios ${ }^{2}$ \\ ${ }^{1}$ Nephrologist, Nephrology Department, Aretaieio Hospital National and Kapodistrian University of Athens, Athens Greece \\ ${ }^{2}$ Panokostas Dimitrios, Nephrology training Nephrology Department, Aretaieio Hospital
}

\begin{abstract}

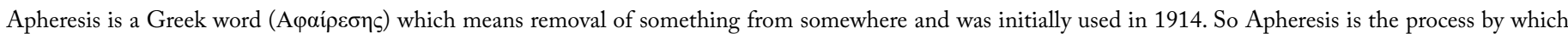
plasma or cellular components are separated from the whole blood. This procedure is performed either on a blood donor, in order to supply the products to patients with different hematological or cancer diseases, or on a patient, alternatively as a therapeutic method. The process may be conducted by centrifugation and we can remove apart from the plasma, white blood cells, red blood cells as well as platelets. Furthermore, the process may be conducted by dialysis through a high permeability dialyzer which specifically removes plasma. Citrates or heparin are used as anticoagulants whereas fluid replacement comprises of albumin or fresh frozen plasma as well as colloidal starch when the procedure is plasma exchange. Complications are related to the vascular access, the anticoagulant or the replacement fluid (frequently with fresh frozen plasma). Now days an increased number of diseases are treated through apheresis procedures and this fact increased the interest of the medical world. Through this paper we focus on therapeutic apheresis principles as they are accepted worldwide, moreover contributing data from our 20 year experience in this field.
\end{abstract}

\section{Introduction}

Therapeutic Apheresis is an accepted treatment worldwide for many autoimmune diseases and refers to an extracorporeal procedure by which blood separating technology is used to remove plasma or abnormal blood cells and plasma components. Historical data provides us the information regarding the idea of Apheresis as a therapeutic method during the archaic era amongst Indian, Egyptian, Greek and Jew population. Fleecing which was performed in the mild age for therapeutic purposes was also a kind of apheresis [1]. Apheresis and plasmapheresis are Greek words which are being used since 1914 by Abel et al [2]. They removed plasma in order to cure uremic dogs .Improvement of their symptoms was observed and the authors reported the whole procedure as apheresis. Ever since this definition was applied in order to mean the removal of something from somewhere (blood). The Abel's experimental trials in 1914 were impossible to be conveyed into clinical practice due to the lack of an appropriate device. Well along into the $20 \mathrm{o}$ century other investigators had the idea to treat diseases removing plasma from the patient $[3,4]$. Years later in 1940 professor E.J Cohn from the Medical School of Harvard, designed a centrifugal device of blood, which was similar to the centrifugal machine of milk (De Laval). Consequently the collection of large amounts of plasma intended for the soldiers during the WWII became possible [5]. Plasma exchange was used for the first time in 1952 by the Adams WS et al, in order to treat a patient diagnosed with multiple myeloma and hyperviscosity [6] In 1965, the engineer G. Jodson in collaboration with the physician E. Freireich and the IBM industry created a centrifugal machine in order to remove white blood cells from a patient with acute leukemic disease (Cancer Inst Houston USA) [7]. Initially the centrifugal machine was used to collect White blood cells and platelets. Finally in 1970 therapeutic apheresis became a reality [8]. Today a number of plasma exchange devices are available and the procedure came to be safe and easy to perform. Plasma exchange (PE) or plasmapheresis, was the first to be used and despite the efforts for more specific treatments it still remains the most frequent procedure.
Apheresis procedure is performed: On a blood donor in order to supply the products in patients with different Hematological or Cancer diseases, or on a patient as a therapeutic method.

Technical modalities: The process is conducted by: centrifugation, by Filtration or by combination of the two modalities (Centifuge Filter Combination ) which may be applied for selective treatments.

Centrifugation: We can separate plasma, white blood cells, red blood cells or platelets. Centrifugation devices are commonly used for blood banking given that they are capable of selective cell removal (cytapheresis) in addition to plasmaferesis. During centrifugation, blood cells are separated by specific gravity based on the different densities of the blood components. There are two types of centrifugation devices, intermittent -flow and continuous -flow.

The intermittent flow method requires a single needle vascular access and is rarely used for therapeutic apheresis. The continuous -flow system requires two venous accesses, one for withdrawal of blood and a second one for its return. This is the most commonly used for therapeutic apheresis. With the continuous -flow devises we sustain a reduced extracorporeal blood volume, significantly shorter procedure time and lesser amount of anticoagulation requirement. Cobe Spectra (Caridian BCT, Lakewood CO) is the most frequently used continuous -flow machine, with the capability to intermittent flow when it's needed. Our 20 year experience with the Cobe Spectra machine provided us with significant therapeutic outcomes lacking any technical problems. Spectra Optia is a new centrifugation system device by Caridian BCT Inc. 2009 which is being used in USA and Europe [9].

Correspondence to: Grapsa Eirini, Nephrology Department, Aretaieio Hospital National and Kapodistrian University of Athens, Athens Greece, Tel: 306972700990; E-mail: grapse@otenet.gr

Received: February 28, 2017; Accepted: March 27, 2017; Published: March 31, 2017 
Filtration: with the use of membrane separators, the process, isolates blood components on the basis of differences in particle size. Regularly plasma is separated from the cellular elements.

Plasma separators (MPS) use membranes with a pore size of 0.6 microns and generally are sufficient in allowing the passage of immune complexes. At the present they can be manufactured in a hollow -fiber and during the past a parallel-plate configuration. One of the most common plasma separator membrane is the Plasma -Flo made by Asahi (Apheresis Technologies Palm Harbor FL). The Asahi plasma flow filter can be used by different devices such as the ACCURA(Baxter Deerfield IL) or DIAPACT (B.Braun Bethlehen PA). Plasauto EZ is also a blood purification device used in extracorporeal circulation therapies in conjunction with Asahi Kasei Kuraray Medical's disposable devices [10].

Plasma exchange with membrane separators needs to be performed at low TMP $(<500 \mathrm{mmHg})$ in order to avoid hemolysis and the blood flow should be greater than $50 \mathrm{ml} / \mathrm{min}$ to avoid clotting. Membrane apheresis is a fast and efficient method where no citrate anticoagulant is required but removal of substances is limited due to sieving coefficient. Furthermore it requires central venous access. Moreover it is unsuitable for treating patients with hyperviscosity syndrome due to paraproteinemia or patients with cryoglobulinemia since the available devices are not efficient enough, in removing large macromolecules.

Selective Apheresis: The efforts for a selective therapy in order to preserve the patient's plasma, created different types of apheresis treatments such as: Plasma adsorption, Double filtration plasma absorption, LDL-apheresis treatment Selectively removal of leukocytes and Extracorporeal photo chemotherapy.

Plasma absorption (pa): In PA, plasma is separated with a plasma separator and flows into a plasma adsorption column. Pathogenic substances are adsorbed and removed due to the affinity between ligands and pathogenic substances. The advantage of this method is that no substitution fluid is required.

Double filtration plasma absorption (DFPP): In DFPP, plasma is separated with a plasma separator and passes through the plasma component separator with a small pore size. Large molecular-weight proteins are discarded and small molecular-weight substances, including valuable albumin, are returned to the patient. A small amount of substitution fluid such as albumin may be added [11].

LDL -Apheresis System: They are a whole blood apheresis machines: The LDL cholesterol, Lp (a) and triglycerides are removed from the blood deprived of the need to separate the blood into red cells and plasma. This means that the treatment is generally shorter in time. There are 2 companies which produce whole blood apheresis technology: Kaneka which manufactures the DX21 device ASAHI KASE and the Fresenius Medical Care which produces the DALI device [12].

Leukocytapheresis: Cellsorba is a leukocytaphersis column used in the treatment of inflammatory bowel diseases. Selectively removes leukocytes implicated in the inflammatory process. As a result it improves the clinical inflammatory status of patients. The regimen involves direct hemoperfusion, in which whole blood is applied to the column for the adsorption of leukocytes onto the nonwoven hydrophilic polypropylene fabric of the column [13]

Extracorporeal photochemotherapy (ECP): is a form of apheresis therapy and involves light-activated treatment of circulating blood cells outside the body (Therakos UVAR XTS Phototheresis System) [14].

\section{Vascular access}

The vascular accesses used are: Pheripheral vein (antecubital), double lumen catheter (subclavian, jugular or femoral), arteriovenous fistula, graft (polytetrafluoroethylene),or permanent double lumen catheter (Broviac Hickman catheter). Peripheral vein (antecubital) is the easiest and safest vascular access once large peripheral veins are available. But frequently in-dwelling vascular access devices are required, when peripheral veins are not available for a continuing flow moreover when the patients need periodic treatment. In addition devices with membrane separation are in need of a central venous catheter as a vascular access. In 1996 we presented data from the TGH Apheresis Unit by which $49 \%$ of the total number of the vascular accesses was peripheral venous and $51 \%$ was central catheters. Thirty three patients presented problems related to the vascular access (catheter thrombosis, poor blood flow or bleeding). One patient faced catheter related sepsis and one patient developed haemopneumothorax. These data suggests a correlation of severe complications to in- dwelling vascular access [15].

We are using peripheral veins more often (75\%) than central venous access (20\%) and scarcely (5\%) permanent access (fistula or graft). We had no severe complications with the central venous catheters apart from poor blood flow due to thrombosis of the catheter. Planning of permanent vascular access should be taken under consideration when chronic PE is needed [16].

\section{Anticoagulation}

Citrate solution and heparin are both used in PE procedures. Centrifugation devices are mostly operated with citrate. Heparin can be used at an initial loading dose of 50 units/Kg following by an infusion rate of 1000 unit per hour. Citrate dextrose (ACD) is used as anticoagulation solution for most therapeutic plasma exchange procedures. Citrate chelates calcium, which is a necessary cofactor in the coagulation cascade, inhibits thrombus formation and platelet aggregation. Bleeding is uncommon with citrate but low plasma ionized calcium commonly occurs $(60 \%-70 \%$ of the overall complications during PE). Symptoms and signs of hypocalcemia must be carefully monitored (acral paresthesias, shivering, twitching, tremors, muscular contractions). Low ionized calcium can provoke fatal arrhythmias in apheresis patients [17]. These complications had be avoided by adding calcium to the replacement solution. Thus ,no complications concerning ionized serum $\mathrm{Ca}++$ were noticed during more than 10.000 procedures.

\section{Replacement solution}

Human albumin (4\% or 5\%) is commonly used and widely accepted as replacement fluid for routine PE, except the Hematological diseases were fresh frozen plasma is used. However clinical experience has demonstrated that the use of colloidal starch (hydroxyethyl starch: HES) as a partial replacement solution is well tolerated, drug interaction is minimal, and it is cost-effective when compared to human albumin. In our unit we used hydroxyethyl starch for more than 10 years in order to achieve cost reduction. No side effects were observed during the procedures. One patient complained about agitation at home and hydroxyethyl starch was stopped [18].

\section{Complications}

The side effects are generally not severe and can be managed easily. They may be related to vascular access, to the procedure or to the anticoagulation. Complications range is $4 \%$ to $25 \%$ with an average of $10 \%$. Data from the TGH Apheresis Unit which was presented at the 
World Apheresis Association $6^{\text {th }}$ International Congress in 1996, shows no complications in 249 patients during 2086 procedures (96\%). Thirty three patients had problems related to vascular access. One or more side effects occurred during 46 procedures in 43 patients. The most common complications were cramps, $(4 \%)$ chills or rigor $(4 \%)$ nausea/ vomiting (3\%), hypotension (1.6\%), hypertension $(0.8 \%)$, headache or dizziness (1.6) fever $(0.8 \%)$ oral paraesthesia ( 0.8$)$ pruritis agitation (1.6) and petechiae formation $(0.4 \%)$. All of these were transient and had little clinical significance. No deaths or life threatening complications occurred $[15,19]$. Our apheresis unit presents a similar complication rate (3\%) in a total or 4500 procedures(14years) and were all transient with out severe or fatal complications [16].

\section{Clinical Applications}

Apheresis procedures are performed for various hematologic, neurological, renal, autoimmune, metabolic, and other indications. But it's true that during the previous years a number of autoimmune diseases have been treated with plasma exchange and in most cases approved no beneficial effect. So, over time the American Society for Apheresis (ASFA) has devoted its considerable expertise towards assessing the state of therapeutic apheresis and were systematically categorized and furthermore clinical guidelines were offered. Beginning with the 2007 ASFA Special Issue (Fourth Edition), the committee has incorporated a systematic review and evidence-based approach in the grading and categorization of indications. The 2007 publication had 5 categories (I-IV and P) according to the indications and the efficacy of the treatment. The categories I and II include diseases for which PE is standard and acceptable either as a primary therapy or a valuable first line therapy or is generally accepted but considered to be supportive or adjunctive to other. The category III diseases are those in which there is a suggestion of benefit for which existing evidence is insufficient either in establishing the efficacy of PE or to clarify the risk /benefit or the cost benefit ratio associated with PE. The category IV indicates disorders about which controlled trials have not shown any benefit or anecdotal reports have been discouraging, which have been carried out in an IRB -approved research protocol. Finally the category P (Pending) includes diseases that can be treated by therapeutic apheresis using devices which are not available in the US and or do not have FDA clearance. So under this classification a number of 53 diseases are included. But if we focus on category I a few number of diseases are included such as Cryoglobulinemia, Thrombotic thrombocytopenic purpura (TTP) Homozygotes familiar Hypercolesteronemia, Neurological diseases: Myasthenia gravis Acute inflammatory demyelinating polyneuropathy, (AGBS), Chronic inflammatory demyelinating polyradiculoneuropathy, (CIDP), Paraproteinemic polyneuropathies, Pediatric autoimmune neuropsychiatric disorders associated with streptococcal infections, Sydenham's chorea, Goodpasture's syndrome, Heart transplant rejection. Following that, every 3 years, new guide lines are published by ASFA according to new experience and diseases could change category [20,21]. In 2011 we presented an abstract in the ASFA annual meeting regarding our experience with 267 patients treated with plasma exchange, of a mean age $65(2,5-80)$ years old using the Cobe Spectra machine. Myasthenia gravis, AGBS, TTP and Heterozygous Familial Hypercolesteronemia were the most common diseases. Only two patients had no response to therapy and the treatment was stopped [16]. Some of the patients needed chronic periodic treatment in order to maintain the results $[22,23]$. During the 20 years of our experience, more than 11000 therapeutic apheresis procedures have been carried out and all the above mentioned diseases have been treated. Patients from all ages, had significant benefit from the treatment. The small rate and the transient of the complications insure the safety of the procedures, when there are appropriate experience and skilled nurses. We started with 40 procedures per year in 1996 and we have reached 800 per year with significant improvement for the majority of the patients.

\section{Conclusion}

Therapeutic Apheresis is an available and safe treatment which helps patients with acute or chronic diseases in reducing symptoms and to improve their quality of life. Knowledge and experience are significant in order to have a rationale use and to minimize the complications.

\section{References}

1. Castiglioni A, Krumbhaar A (1941) History of Medicine New York Allfred A Knopf 77-197.

2. Abel J, Rowntree G, Turner B (1914) Plasma removal with return of corpuscles. $J$ Pharmacol Exp Ther 5: 625-641.

3. Kalman B (1991) Early Health and Medicine. Toronto ,New York Grabtree Publishing Co 8 .

4. Shumak KH, Rock GA (1984) Therapeutic plasma exchange. N Engl J Med 310: 762 771. [Crossref]

5. Millward BL, Hoeltge GA (1982) The historical development of automated hemapheresis. J Clin Apher 1: 25-32. [Crossref]

6. Adams WS, Blahd WH, Bassett SH (1952) A method of human plasmapheresis. Proc Soc Exp Biol Med 80: 377-379. [Crossref]

7. Freireich EJ, Judson G, Levin RH (1965) Separation and collection of leukocytes. Cancer Res 25: 1516-1520. [Crossref]

8. Lockwood CM, Rees AJ, Pearson TA, Evans DJ, Peters DK, et al. (1976) Immunosuppression and plasma exchange in the treatment of Goodpasture's syndrome Lancet. 1:7 11-715.

9. Kaplan AA (2013) Therapeutic plasma exchange: a technical and operational review. $J$ Clin Apher 28: 3-10. [Crossref]

10. Hirata N, Shizume Y, Shirokaze J, Suemitsu J, Yoshida H, et al. (2003) Plasma separator Plasmaflo OP. Ther Apher Dial 7: 64-68. [Crossref]

11. Julius U, Parhofer KG, Heibges A, Kurz S, Klingel R, et al. (2007) Dextran-sulfateadsorption of atherosclerotic lipoproteins from whole blood or separated plasma for lipid-apheresis--comparison of performance characteristics with DALI and Lipidfiltration. J Clin Apher 22: 215-223. [Crossref]

12. Bambauer R, Bambauer C, Lehmann B, Latza R, Schiel R (2012) LDL-apheresis technical and clinical aspects. ScientificWorldJournal 2012: 314283. [Crossref]

13. Suemitsu J, Yoshida M, Yamawaki N, Yamashita Y (1998) Leukocytapheresis therapy by extracorporeal circulation using a leukocyte removal filter. Ther Apher 2: 31-36. [Crossref]

14. Knobler R, Berlin G, Calzavara-Pinton P, Greinix H, Jaksch P, et al. (2014) Guidelines on the use of extracorporeal photopheresis. J Eur Acad Dermatol Venereol 28 Suppl 1: 1-37. [Crossref]

15. Grapsa E ,Sutton D. WAA Congress 1996 Florence Italy Abstract

16. Grapsa E, Mpakirzi T, Papaioannou N, Geroggianni R, Pantelias K, et al. (2011) Clin Apher 26: 91.

17. Lee G, Arepally GM (2012) Anticoagulation techniques in apheresis: from heparin to citrate and beyond. J Clin Apher 27: 117-125. [Crossref]

18. Ward DM (2011) Conventional apheresis therapies: a review. J Clin Apher 26: 230238. [Crossref]

19. Mokrzycki MH, Balogun RA (2011) Therapeutic apheresis: a review of complications and recommendations for prevention and management. J Clin Apher 26: 243-248. [Crossref]

20. Schwartz J, Winters JL, Padmanabhan A, Balogun RA, Delaney M, et al. (2013) Guidelines on the use of therapeutic apheresis in clinical practice-evidence-based approach from the Writing Committee of the American Society for Apheresis: the sixth special issue. J Clin Apher 28: 145-284.

21. Schwartz J, Padmanabhan A, Aqui N, Balogun RA, Connelly-Smith L, et al. (2016) Guidelines on the Use of Therapeutic Apheresis in Clinical Practice-Evidence-Based Approach from the Writing Committee of the American Society for Apheresis: The Seventh Special Issue. J Clin Apher 31: 149-162. [Crossref] 
22. Grapsa E, Triantafyllou N, Rombos A, Lagouranis A, Dimopoulos MA (2008) Therapeutic plasma exchange combined with immunomodulating agents in secondary progressive multiple sclerosis patients. Ther Apher Dial 12: 105-108. [Crossref]
23. N. Triantafyllou, Grapsa EI, Kararizou E, Psimenou E, Lagguranis A, et al. (2009) Periodic therapeutic plasma exchange in patients with moderate to severe chronic myasthenia gravis non-responsive to immunosuppressive agents: an eight-year followup. Ther Apher Dial 13: 174-178. [Crossref]

Copyright: (C2017 Eirini G. This is an open-access article distributed under the terms of the Creative Commons Attribution License, which permits unrestricted use, distribution, and reproduction in any medium, provided the original author and source are credited. 